\title{
Evaluation and Management of In-stent Original Low-density Area on CTA after Carotid Artery Stenting at Subacute Phase
}

Osamu Suzuki, Shinnosuke Hattori, Yuichi Ito, and Masaaki Kimura

Objective: We examined the prognosis and background factors associated with in-stent low-density areas (LDAs) on CTA in the subacute phase after carotid artery stenting (CAS).

Materials and Methods: Among 175 lesions for which CAS was performed between April 2008 and September 2016, 100 lesions for which CTA were conducted in the subacute phase after CAS were included for retrospective analysis.

Results: LDAs were observed in $36(36.0 \%)$ of the 100 lesions. All patients received conservative medical treatment. There was no patient with new neurologic symptoms in the subacute phase. In most lesions, peak stenosis rate was observed relatively early, which gradually declined after 1 week. LDAs were observed and the stenosis rate was relatively high in all patients with plaque protrusion immediately after CAS. The use of open-cell stents significantly increased the formation of LDAs. Most LDAs were consistent at the level of the previous most stenotic sites.

Conclusion: LDAs in the subacute phase after CAS were relatively frequent. In this study, the course was asymptomatic in all patients, and remission was gradually achieved. However, LDAs may have been associated with serious ischemic complications in the subacute phase, as reported in the literature. Therefore, medical treatment, involving intensive antithrombotic therapy, and careful follow-up should be considered.

Keywords \ carotid artery stenting, subacute phase, low-density area, computed tomography angiography

\section{Introduction}

Carotid artery stenting (CAS) has been increasingly used as a common procedure after the Stenting and Angioplasty with Protection in Patients at High Risk for Endarterectomy study (SAPPHIRE) and the Carotid Revascularization Endarterectomy vs. Stenting Trial (CREST) demonstrated its usefulness. ${ }^{1,2}$ However, the incidence of perioperative ischemic complications is higher than in patients undergoing carotid endarterectomy (CEA), as indicated by the CREST and the International Carotid Stenting Study. 2,3$)$

Department of Neurosurgery, Nagoya Ekisaikai Hospital, Nagoya, Aichi, Japan

Received: February 28, 2017; Accepted: October 16, 2017 Corresponding author: Osamu Suzuki. Department of Neurosurgery, Nagoya Ekisaikai Hospital, 4-66 Shonen-cho, Nakagawa-ku, Nagoya, Aichi 454-8502, Japan

Email: osuzukijp@yahoo.co.jp

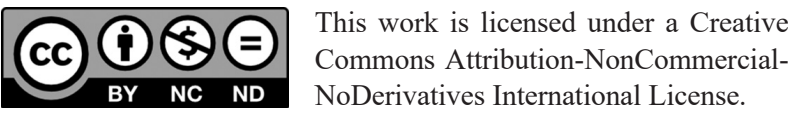

(C)2018 The Japanese Society for Neuroendovascular Therapy
Several studies reported that an in-stent low-density area (LDA) was often observed on CTA in the subacute phase after CAS, and that the extent of most LDAs was reduced with medication. ${ }^{4-8)}$ On the other hand, some studies reported ischemic complications in the subacute phase of $\mathrm{CAS}^{6,8-13)}$ as an important issue.

The incidence of perioperative complications related to CAS might be reduced by identification of causes and time course of LDA, and development of appropriate treatment and follow-up methods may improve outcomes of CAS. At our hospital, treatment methods are chosen in accordance with the degree of stenosis in patients with an LDA detected on CTA in the subacute phase after CAS. In this study, we retrospectively examined the incidence, time course, background factors, and imaging characteristics of LDAs in patients who underwent CAS at our hospital. We also reviewed treatment and follow-up methods.

\section{Materials and Methods}

Among a total of 175 lesions in 159 patients who underwent CAS at our hospital between April 2008 and September 2016, 
100 lesions in 93 patients who underwent CTA in the subacute phase after CAS were included in this retrospective study. According to our hospital protocols, CTA is performed for postoperative assessment in patients with an estimated glomerular filtration rate (eGFR) of $\geq 50 \mathrm{~mL} /$ $\min / 1.73 \mathrm{~m}^{2}$, whereas ultrasonography is performed in those with an eGFR of $<50 \mathrm{~mL} / \mathrm{min} / 1.73 \mathrm{~m}^{2}$. In this study cohort, a total of 75 lesions were excluded, as CTA was not performed, including 51 lesions with their pre-CAS eGFR $<50 \mathrm{~mL} / \mathrm{min} / 1.73 \mathrm{~m}^{2}$. In 14 lesions, eGFR transiently dropped to $<50 \mathrm{~mL} / \mathrm{min} / 1.73 \mathrm{~m}^{2}$ after CAS, and CTA was not examined. CTA was not performed in seven lesions due to complications such as heart failure and cholesterol embolism. Additionally, CAS could not be accomplished in three lesions and thus, CTA was discontinued.

The study cohort comprised 82 males and 11 females, with a mean age of 71.5 years. There were 58 symptomatic and 42 asymptomatic lesions, and 7 patients had bilateral lesions. Sites of stenosis consisted of the origin of the internal carotid artery (ICA) in 89 lesions and common carotid artery (CCA) in 11 lesions.

One hundred $\mathrm{mg} /$ day of aspirin and $75 \mathrm{mg} /$ day of clopidogrel were administered at least 7 days before CAS. Additionally, $200 \mathrm{mg}$ /day of cilostazol was initiated 1 day before CAS. CAS was performed under local anesthesia by transfemoral or transbrachial approach. After sheath placement, heparin was intravenously administered to achieve an activated clotting time twoto three-fold. The distal protection method with a PercuSurge GuardWire Plus (Medtronic, Minneapolis, MN, USA) was usually adopted; however, distal filter or proximal protection methods were used in some patients. Predilatation was conducted using a balloon catheter with a diameter of $3 \mathrm{~mm}$ in all lesions, and postdilatation was performed using a balloon that was $0.5-1.0 \mathrm{~mm}$ smaller than the diameter of the ICA just distal to the stenosis. A closed-cell stent was used as the first choice; however, an open-cell stent was used primarily for a tortuous lesion. Heparin was routinely administered at 15000 units/day for 3 days following CAS. Aspirin, clopidogrel, and cilostazol were orally administered for 5 days after CAS, and aspirin and clopidogrel were administered thereafter for 3 months. Subsequently, the oral administration of a single drug, aspirin or clopidogrel, was continued.

MRI including diffusion-weighted images (DWIs) was performed 3-6 days after CAS. CTA was examined 5-7 days after CAS. In-stent restenosis was evaluated

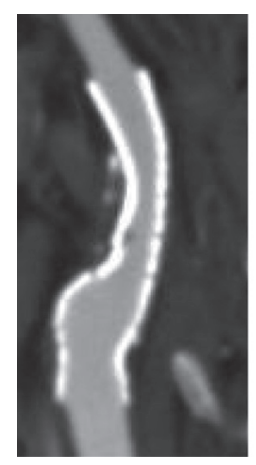

A

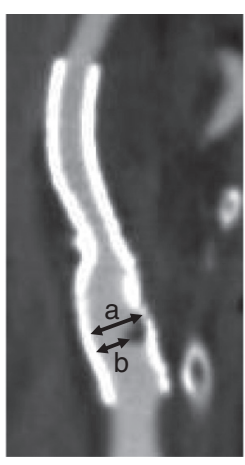

B

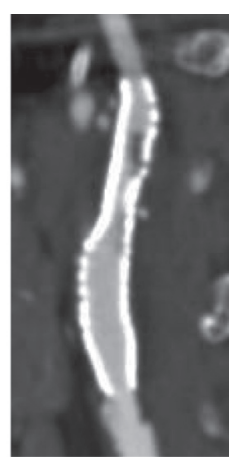

$\mathrm{C}$
Fig. 1 Classification of in-stent LDA on CTA at subacute phase. LDA is classified into three groups depending on the degree of stenosis at CTA. (A) Stenosis rate is $<20 \%$, (B) stenosis rate is between $20 \%$ and $50 \%$, (C) stenosis rate is $\geq 50 \%$, stenosis rate $=(a-b) / a \times 100 \%$. LDA: low-density area

using CTA or ultrasonography at 3, 6, and 12 months after CAS, and annually thereafter.

For CTA, an Aquilion 64 (64 slices) or Aquilion PRIME (80 slices) multidetector row CT (MDCT; Toshiba Medical Systems, Ohtawara, Japan) was used. Images were reconstructed using a 3D Work Station from Ziostation (Ziosoft, Tokyo, Japan). Imaging conditions were established as follows: tubal voltage, $120 \mathrm{kV}$; tubal current, 300-350 mA; scan time, 0.5 seconds/rotation; slice thickness, $0.5 \mathrm{~mm}$; and helical pitch, 51. Nonionic iodine contrast medium at $370 \mathrm{mgI}$ was infused at a rate of $4.0 \mathrm{~mL} / \mathrm{sec}$ after securing a venous route using a $20-\mathrm{G}$ indwelling needle. Basically, the infusion volume was $50 \mathrm{~mL}$, and it was adjusted in accordance with the body weight, if necessary. The Real prep method was adopted, and imaging was manually started when an increase in CT value of the CCA could be visually confirmed. Furthermore, slice thickness for image reconstruction was established as $0.5 \mathrm{~mm}$, and slice interval was $0.3 \mathrm{~mm}$.

LDAs were evaluated by preparing a vertical curved multiplanar reconstruction (MPR) to the course of the blood vessel into which a stent was placed using the $3 \mathrm{D}$ Work Station. The window level was adequately adjusted, and in-stent low-attenuation areas were defined as LDAs. The stenosis rate was calculated by measuring stent lumen diameter and length of low-attenuation area at the level of the maximum stenotic segment (Fig. 1B). Cases were classified as no stenosis, stenosis $<20 \%$, stenosis between $20 \%$ and $50 \%$, and stenosis $\geq 50 \%$ (Fig. 1). In cases with a stenosis rate of $<20 \%$, postoperative antithrombotic therapy and follow-up were performed as 


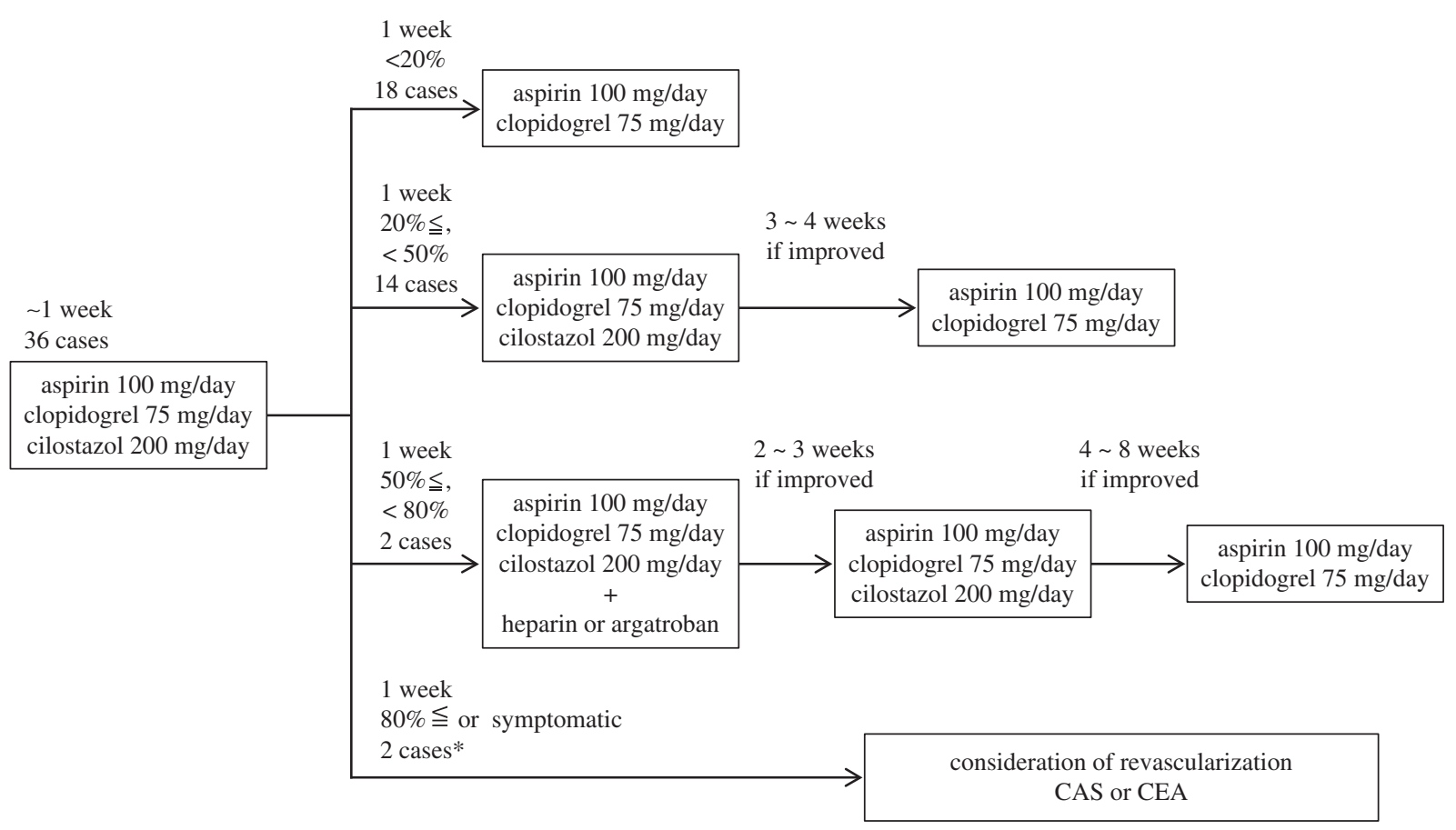

Fig. 2 Strategy of management for LDA on CTA at subacute phase. CAS: carotid artery stenting; CEA: carotid endarterectomy; LDA: low-density area; ${ }^{*} 1$ case: asymptomatic occlusion, 1 case: conservative therapy with resolution of the severe stenosis

scheduled. In cases with a stenosis between $20 \%$ and $50 \%, 200 \mathrm{mg} /$ day cilostazol was additionally administered $\geq 6$ days after CAS (triple antiplatelet therapy) until further examination 3-4 weeks after. In cases with a stenosis rate of $\geq 50 \%$, heparin or argatroban was administered in addition to the continued triple antiplatelet therapy until next evaluation 1-2 weeks after. Subsequently, triple antiplatelet therapy was continued until LDA was declined. In patients with symptomatic stenosis and in those with a stenosis rate of $\geq 80 \%$, additional revascularization by endovascular surgery or CEA was considered (Fig. 2).

The presence of LDAs, the stenosis rate, clinical course, perioperative ischemic complications, and DWIs between LDA(+) and LDA(-) groups were analyzed. Furthermore, we compared age, sex, presence of hypertension, diabetes and hyperlipidemia, and symptomatic or asymptomatic stenosis between the two groups. Patients receiving drug therapy for hypertension, diabetes, and/or hyperlipidemia before CAS were classified as those with a history of hypertension, diabetes, and/or hyperlipidemia. We analyzed the relationships with the presence of calcifications, ulcers, unstable plaques, and length of stenosis and degree of stenosis in both groups. The relationship of lesions at the ICA origin with the branching angle of the ICA was also investigated. Plaque properties were evaluated using the MRI black blood method (MRI BB). Plaques with a signal intensity higher than that of the sternocleidomastoid muscle on either or both T1- and T2-weighted images were defined as unstable plaques. ${ }^{14)}$ To determine the branching angle of the ICA, the angle between the longitudinal axis of the CCA and that of the ICA immediately after branching was measured on DSA. ${ }^{15)}$

As procedure-associated factors, we analyzed the relationships with diameter of balloon for postdilatation, residual stenosis immediately after CAS, closed-/open-cell stents, and stent length. In cases where DSA showed plaque protrusion immediately after CAS, we investigated its relationship with the presence of an LDA and its location. Among patients with $\geq 20 \%$ stenosis, sequential changes of stenosis were observed by serial CTA.

For statistical analysis, the EZR software (version 1.31; Saitama Medical Center, Jichi Medical University, Saitama, Japan) was used. Data were expressed as means \pm standard deviation. Continuous variables were compared between the two groups using Student t-test or the Mann-Whitney U-test. For categorical comparisons, univariate analysis was performed using the chi-square test or Fisher exact test. Logistic regression analysis was performed including factors with a $p$ value $<0.15$ on univariate analysis. $\mathrm{p}$ values $<0.05$ were considered as statistically significant. 


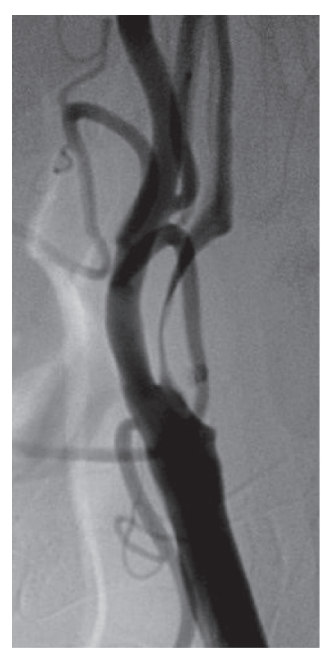

A

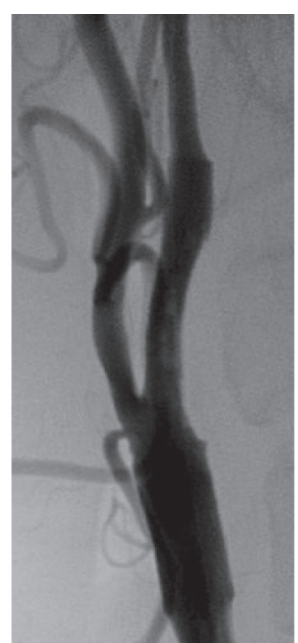

B

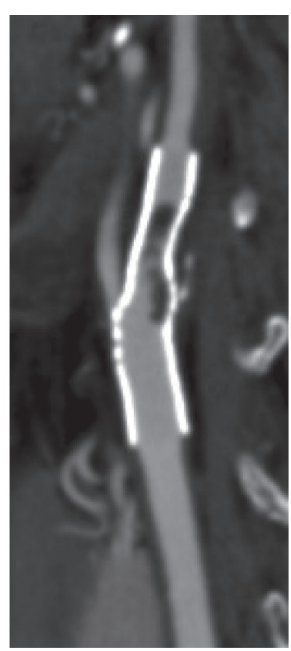

$\mathrm{C}$

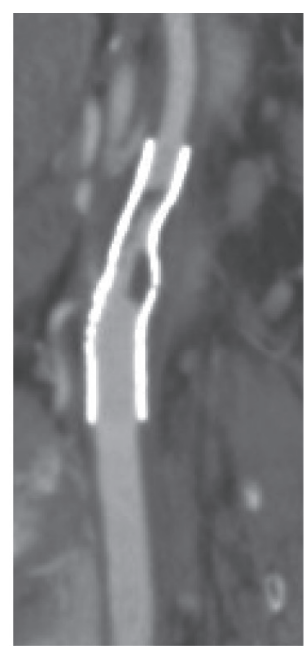

D

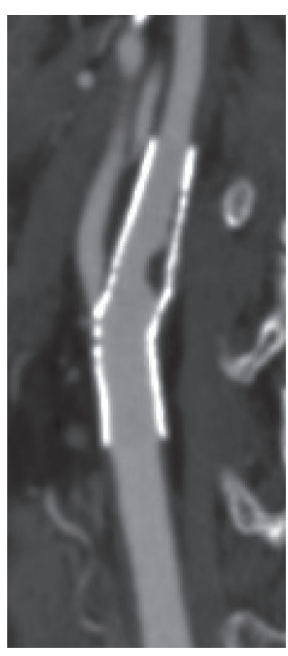

$\mathrm{E}$

Fig. 3 A case of severe stenosis treated by medical treatment alone. (A) A lateral view of preprocedural carotid angiogram. (B) A lateral view of carotid angiogram immediately after CAS. (C) CTA obtained 7 days after CAS showed severe stenosis. This patient was treated by medical treatment alone. (D) 12 days after CAS. (E) 4 months after CAS, CTAs showed LDA decreased gradually without any symptoms. CAS: carotid artery stenting; LDA: low-density area

\section{Results}

Among 100 lesions in 93 patients, postoperative CTA revealed LDAs in 36 lesions (36\%). Among these, $<20 \%$, $20 \%-50 \%$, and $\geq 50 \%$ stenosis were observed in $18(18 \%)$, $14(14 \%)$, and $4(4 \%)$ lesions, respectively. In one of the four lesions with $\geq 50 \%$ stenosis, asymptomatic complete occlusion was observed. There was no patient with neurologic symptoms during the course, except for one patient in the $\operatorname{LDA}(+)$ group who experienced transient ischemia in the vertebro-basilar artery territory immediately after CAS. Postoperative DWIs showed high-signal intensity in the territory of treatment-associated area in $17(48.6 \%)$ of the 35 lesions in the LDA(+) group and in $16(25.0 \%)$ of the 64 lesions in the $\operatorname{LDA}(-)$ group; the rate of positive lesions on DWIs was significantly higher in the LDA(+) group ( $p=0.048$; odds ratio, $2.64,95 \%$ confidence interval, 1.02-6.86).

In the LDA(+) group, $\geq 80 \%$ stenosis was observed in one patient, for whom a second revascularization session was indicated. However, informed consent could not be obtained from this patient, and medical treatment by intensive antithrombotic therapy was continued. As a result, there were no symptoms, and the LDA gradually reduced in size during follow-up (Fig. 3). No patient underwent additional revascularization in the subacute phase in either group. All patients in the $\operatorname{LDA}(+)$ group received medical treatment. A follow-up CTA was obtained in 94, 85, and 75 of the 100 lesions after 3, 6, and 12 months, respectively. No patient developed cerebral infarction in the chronic phase, but a stent-in-stent procedure for marked asymptomatic in-stent restenosis was additionally performed in one patient in each group after 7 and 16 months, respectively.

The comparison of the results between the two groups is shown in Table 1. There were no significant differences in any of the background characteristics including age, sex, and presence of hypertension, hyperlipidemia, and/or diabetes between the two groups. LDAs were slightly more frequent in those with ulcerative lesions or unstable plaques; however, there was no significant difference. The rate of LDA appearance was significantly higher in cases where an open-cell stent was used. In addition, there were no significant differences in the presence of calcification, length of stenosis, preoperative stenosis rate, postoperative stenosis rate, size of the balloon used for postdilatation, stent length, and branching angle of ICA between the two groups.

LDAs appeared at the site of the maximum stenosis before CAS in 31 (86.1\%) of the 36 lesions. Of these, LDAs appeared on the dorsal site of the ICA origin in $20(55.6 \%)$ lesions (Fig. 4A and 4B), while other 11 lesions (30.1\%) appeared on sites other than dorsal (Fig. 4C and 4D). LDAs appeared at sites other than the maximum stenotic sites in 4 (11.1\%) lesions (Fig. 4E and $\mathbf{4 F}$ ). DSA obtained immediately after CAS confirmed plaque protrusion in 


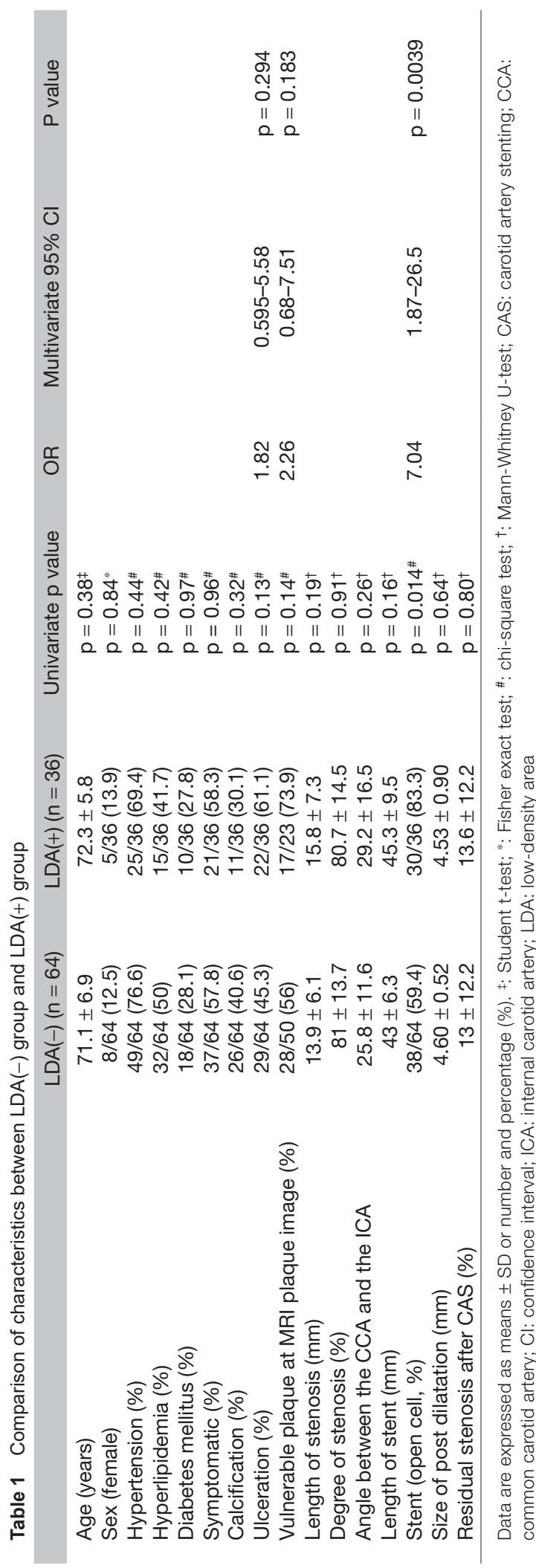

eight lesions. In these cases, LDAs were developed in the same sites as plaque protrusion (Fig. 5). $<20 \%, 20 \%-50 \%$, and $\geq 50 \%$ stenosis were found in two, five, and one lesion, respectively, which were relatively high incidence. Among patients with $\geq 20 \%$ LDAs, the course of those patients who could be serially followed-up using CTA is shown in Fig. 6. In most of these patients, the stenosis rate reached a peak during the first CTA follow-up, and stenosis gradually declined after 1 week, following a stable course.

\section{Discussion}

Several studies reported that in-stent LDAs were detected on CTA in the subacute phase after CAS in 5\%-43\% of patients. ${ }^{4-8)}$ The results of the present study also revealed LDAs in $36 \%$ of the lesions. In previous studies, almost all cases with LDAs were asymptomatic and gradually reduced in most patients, but some patients became symptomatic. ${ }^{6,8)}$ In the present study, there were no patients with symptomatic in-stent LDAs, including the one patient with $\geq 80 \%$ stenosis. However, there are many case reports of the appearance of serious ischemic symptoms in the subacute phase after CAS or additional revascularization needed due to repeated ischemic attacks. ${ }^{9-13,16-20)}$ LDAs on CTA in the subacute phase may be associated with these ischemic complications. Appropriate treatment based on early detection of LDA on CTA might decrease the incidence of perioperative complications, consequently improving the results of CAS.

Symptomatic lesions, ulcers, unstable plaques, long stents used, and stent type were previously reported as factors associated with LDA appearance. ${ }^{4-8)}$ In the present study, the use of an open-cell stent was significantly associated with the appearance of LDAs. Jongen et al. ${ }^{4}$ ) reported that nitinol stents significantly induced formation of LDAs compared with stainless stents and suggested that differences in length and shape of stents rather than material-related differences might be associated with this phenomenon. LDA appearance after the use of open-cell stents might be associated with frequent plaque protrusion due to a large free cell area. Furthermore, the presence of ulcers or unstable plaques was associated with LDAs, but there was no significant difference. Conversely, the rate of patients with ischemic lesions on DWIs after CAS was significantly higher in the $\operatorname{LDA}(+)$ group than in the LDA(-) group.

The most frequent LDA location was the site of the maximum stenosis, accounting for $31(86.1 \%)$ of the 


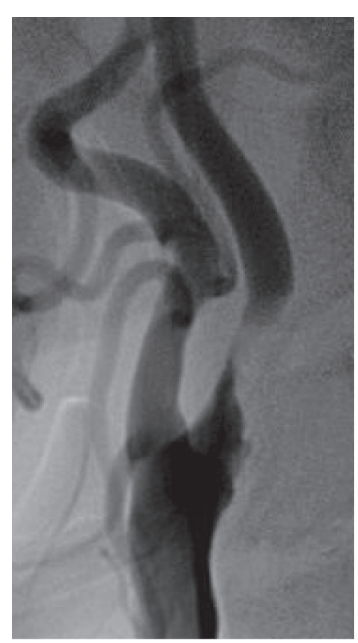

A

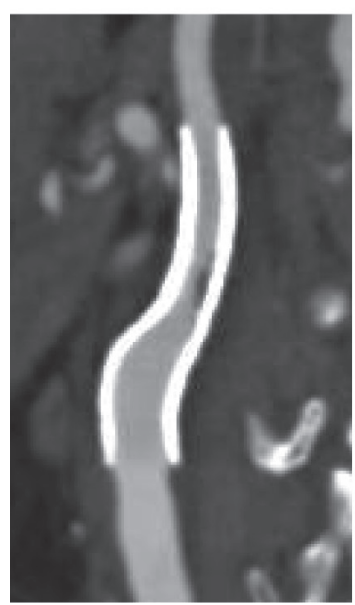

B

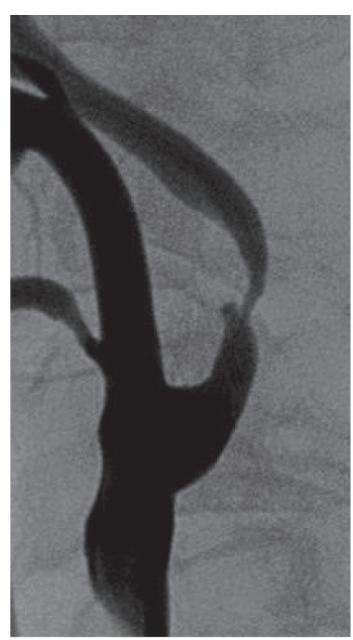

C

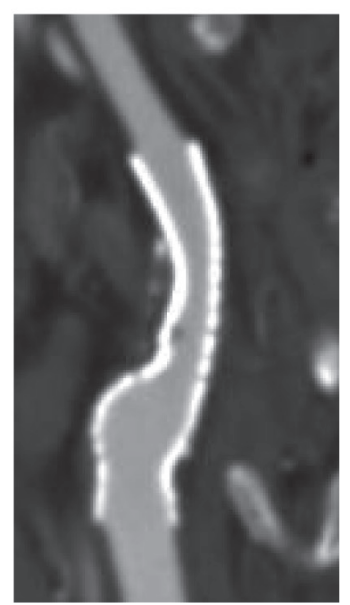

D

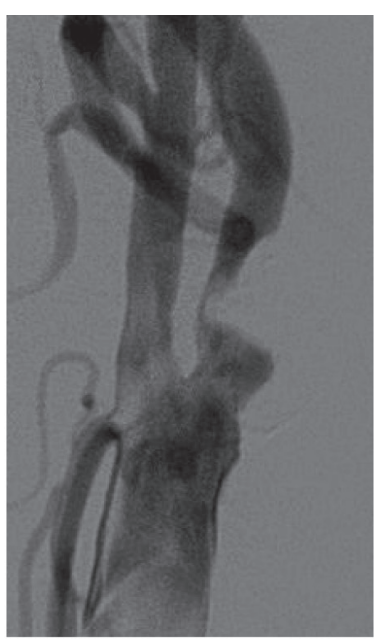

E

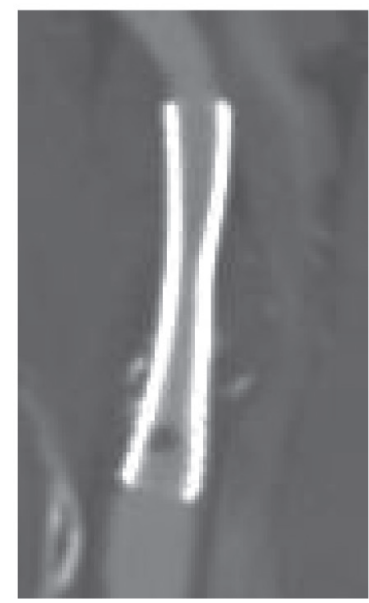

F

Fig. 4 Location of LDA on CTA at subacute phase. (A) A lateral view of preprocedural carotid angiogram. (B) CTA of post-CAS showing LDA at the dorsal site of the proximal ICA. (C) A lateral view of preprocedural carotid angiogram. (D) CTA of post-CAS showing LDA at the maximum preprocedural stenosis, but not at the dorsal site of the proximal ICA. (E) A lateral view of preprocedural carotid angiogram. (F) CTA of post-CAS showing LDA which is not located at the stenotic site. CAS: carotid artery stenting; ICA: internal carotid artery; LDA: low-density area

36 lesions. Previous studies reported that most LDAs were located on the dorsal site of the ICA origin. ${ }^{5,6)}$ Plaque volume and turbulent flow due to the vessel angle may induce LDA. However, the results of the present study revealed that LDAs on the dorsal site of the ICA origin accounted for $55.6 \%$ of the cases, and LDAs at other sites were not rare. Furthermore, examination of the relationship with the branching angle of the ICA origin determined that there was no significant difference between the two groups. Many LDAs appeared at the site of the maximum stenosis before CAS, and LDAs could occur at sites other than the dorsal site of the ICA origin or in straight lesions.
Few studies have pathologically investigated stenosis in the subacute phase. Setacci et al. reported that a thrombus was adhered to the site of plaque protrusion from the stent strut. ${ }^{12)}$ Ozaki et al. also reported a similar finding. ${ }^{8)}$ Conversely, several studies showed that there was no thrombus formation at the site of plaque protrusion. ${ }^{16,17)}$ Thrombi may have been scattered in the latter case. Nonetheless, development of LDA might be related to plaque protrusion from the stent strut. In our cohort, LDAs were observed at the same site with plaque protrusion in all patients that was observed immediately after CAS, and the stenosis rate of patients with plaque protrusion was high in most patients. This finding suggests that LDAs might 


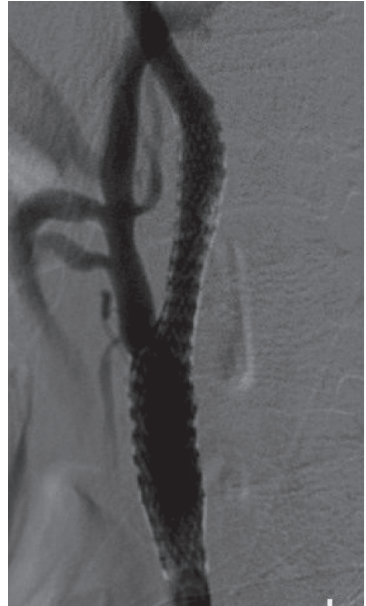

A

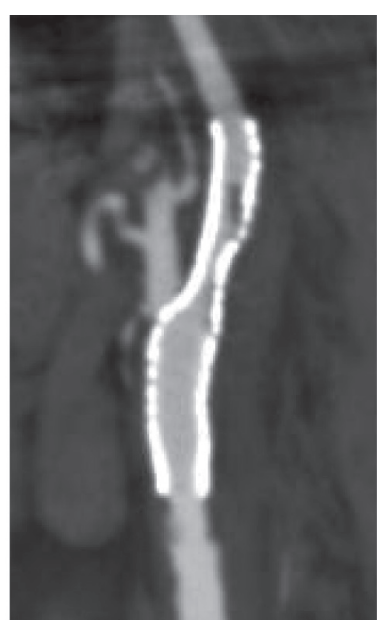

B
Fig. 5 Association of a plaque protrusion immediately after CAS and LDA on CTA at subacute phase. (A) A lateral view of carotid angiogram immediately after CAS. The plaque protrusion was seen at the dorsal site of the ICA. (B) CTA of post-CAS showing LDA at the same location. CAS: carotid artery stenting; ICA: internal carotid artery; LDA: low-density area

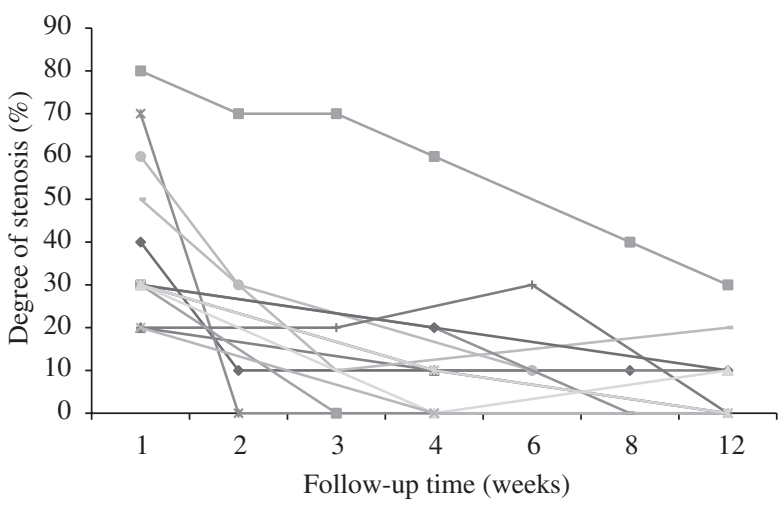

Fig. 6 The sequential changes of LDA on CTA at subacute phase. Those of cases with $>20 \%$ stenosis followed by CTA. In all cases, the maximum degree of stenoses was found at the first follow-up evaluation, and then, the degree of stenoses was gradually decreased. LDA: low-density area

deteriorate by exacerbation of thrombus adhesion to a plaque protrusion from a stent into the lumen. LDAs frequently developed at the site of the maximum stenosis, i.e., the most plaque-abundant site; they also were more frequent in cases where an open-cell stent was used. This is consistent with the results of pathologic studies.

Concerning the timing of LDA appearance, Watarai et al. ${ }^{5)}$ reported that peak LDA appearance was observed on the first follow-up 2 weeks after CAS and that LDAs gradually declined in the subsequent 12 weeks in most patients. In the present study, the first examination was performed 5-7 days after CAS, and the stenosis rate was the highest on the first examination in most patients, and stenosis then gradually decreased after 1 week suggesting that most LDAs appear in a few days and reach a maximum size within 1 week. Therefore, the first examination after CAS should be performed earlier.

Concerning treatment of LDA, many studies indicated the significance of medical treatment, especially antithrombotic therapy. ${ }^{4-13)}$ In a review of stent-related thrombosis after CAS, Moulakakis et al. emphasized that antiplatelet drugs were important for its prevention. They reported that etiological factors for thrombus formation included insufficient administration of antiplatelet drugs, drug resistance, inherent or acquired thrombotic disorders including cancer, and discontinuation of antiplatelet drugs. ${ }^{9)}$ Furthermore, Kobayashi et al. reported a patient with paresis that occurred 7 days after CAS who did not show improvement despite combination therapy with aspirin, clopidogrel, cilostazol, and an anticoagulant; however, switching from clopidogrel to prasugrel led to remission. They speculated that the patient might have resistance to clopidogrel, whereas prasugrel might have exhibited antiplatelet action. ${ }^{20)}$ Several studies indicated that $2 \%-21 \%$ of patients resisted to aspirin, whereas resistance to clopidogrel was $43 \%-65 \%$. Specifically, resistance to clopidogrel might be more closely associated with thrombosis after CAS. ${ }^{21,22)}$ Furthermore, a study indicated that additional administration of cilostazol in clopidogrelresistant patients reduced platelet aggregation, significantly decreasing the incidence of new ischemic lesions on DWIs after CAS. ${ }^{23}$ In the present study, triple antiplatelet therapy was administered in the perioperative phase, which might have significantly inhibited thrombus formation even with the appearance of LDA after CAS, contributing to the absence of symptomatic lesions. However, platelet aggregation monitoring was not conducted, and the relationship between platelet aggregation and LDA formation should be investigated in future studies.

In the present study, treatment involving intensive antithrombotic therapy was performed according to the extent of stenosis in cases with LDAs (Fig. 2). As a result, remission was achieved relatively early in all patients, excluding those with occlusion, and there were no symptomatic patients in the subacute phase. This finding suggests the importance of antithrombotic therapy including antiplatelet drugs. However, Kurisu et al. performed an emergency stent-in-stent procedure for symptomatic subacute in-stent thrombosis, which occurred 7 days after CAS, resulting a 
favorable outcome without sequelae. They indicated that prompt endovascular treatment was effective for symptomatic thrombosis. ${ }^{19)}$ In contrast, Ishihara et al. conducted additional endovascular treatment and intensive antithrombotic therapy for in-stent thrombosis 5 days after CAS; however, cerebral hemorrhage finally occurred, resulting an unfavorable outcome. They stated that surgical procedures, including CEA, must be considered, as long-term intensive antithrombotic therapy increased the risk of hemorrhagic complications. ${ }^{11)}$ It is not always easy to determine the timing of additional revascularization and selection of treatment modality. However, early additional revascularization, including CEA, should be considered in patients with marked stenosis, those who do not improve with medical treatment, and those with symptomatic thrombosis.

\section{Conclusion}

LDAs on CTA in the subacute phase after CAS are not rare and may appear relatively early. The use of open-cell stents was associated with formation of LDAs. Most LDAs were consistent with the site of the maximum stenosis before CAS. In all patients, medical treatment led to remission, and there was no symptomatic lesion in any patient. Appropriate follow-up and antithrombotic therapy including antiplatelet drugs might be important for LDAs in the subacute phase after CAS.

\section{Disclosure Statement}

All authors completed conflict of interest (COI) selfreporting to the Japan Neurosurgical Society. There is no COI to be disclosed regarding this article.

\section{References}

1) Yadav JS, Wholey MH, Kuntz RE, et al: Protected carotidartery stenting versus endarterectomy in high-risk patients. N Engl J Med 2004; 351: 1493-1501.

2) Brott TG, Hobson RW, Howard G, et al: Stenting versus endarterectomy for treatment of carotid-artery stenosis. N Engl J Med 2010; 363: 11-23.

3) International Carotid Stenting Study investigators, Ederle J, Dobson J, et al: Carotid artery stenting compared with endarterectomy in patients with symptomatic carotid stenosis (International carotid stenting study): an interim analysis of a randomised controlled trial. Lancet 2010; 375: 985-997.
4) Jongen LM, Hendrikse J, Waaijer A, et al: Frequency and consequences of early in-stent lesions after carotid artery stent placement. J Vasc Interv Radiol 2009; 20: 573-579.

5) Watarai H, Kaku Y, Yamada M, et al: Follow-up study on in-stent thrombosis after carotid stenting using multidetector CT angiography. Neuroradiology 2009; 51: 243-251.

6) Hashimura N, Mutoh T, Matsuda K, et al: Evaluation and management of plaque protrusion or thrombus following carotid artery stenting. Neurol Med Chir (Tokyo) 2015; 55: 149-154.

7) Okahara M, Kiyosue H, Kashiwagi J, et al: Small in-stent low density on CT angiography after carotid artery stenting. Interv Neuroradiol 2008; 14: 41-46.

8) Ozaki S, Tagawa M, Matsumoto S, et al: [Pathogenesis of in-stent thrombosis after carotid artery stenting]. No Shinkei Geka 2014; 42: 1009-1017. (in Japanese)

9) Moulakakis KG, Mylonas SN, Lazaris A, et al: Acute carotid stent thrombosis: a comprehensive review. Vasc Endovascular Surg 2016; 50: 511-521.

10) Takigawa T, Matsumaru $Y$, Kubo $T$, et al: Recurrent subacute in-stent restenosis after carotid artery stenting due to plaque protrusion. Neurol Med Chir (Tokyo) 2009; 49: 413-417.

11) Ishihara $H$, Ishihara $S$, Kato $H$, et al: A case of repeated acute phase in-stent thrombosis following carotid artery stenting: case report. JNET 2011; 5: 188-194. (in Japanese)

12) Setacci C, de Donato G, Setacci F, et al: Surgical management of acute carotid thrombosis after carotid stenting: a report of three cases. J Vasc Surg 2005; 42: 993-996.

13) Naito T, Miyachi S, Izumi T, et al: [Rescue stenting for subacute thrombosis after carotid stenting -report of 2 cases]. Jpn J stroke 2010; 32: 427-433. (in Japanese)

14) Inukai $C$, Inukai $T$, Matsuo $N$, et al: [Evaluation of carotid artery plaques by black blood magnetic resonance imaging and contrast enhanced computed tomography: correlation of the two methods]. Jpn J Stroke 2010; 32: 229-235. (in Japanese)

15) Daitoku S, Yuasa T, Tsunenari H, et al: Angle between the common and internal carotid arteries detected by ultrasound is related to intima-media thickness among those with atherosclerotic disease. Environ Health Prev Med 2015; 20: 216-223.

16) Ferrero E, Ferri M, Viazzo A, et al: Carotid stent removal of symptomatic plaque protrusion after carotid angioplasty stenting. Interact Cardiovasc Thorac Surg 2010; 11: 254-256.

17) Chaturvedi S, Sohrab S, Tselis A: Carotid stent thrombosis: report of 2 fatal cases. Stroke 2001; 32: 2700-2702. 
18) Masuo O, Terada T, Matsuda Y, et al: Successful recanalization by in-stent percutaneous transluminal angioplasty with distal protection for acute carotid stent thrombosis. Neurol Med Chir (Tokyo) 2006; 46: 495-499.

19) Kurisu K, Manabe H, Ihara T: [Case of symptomatic subacute in-stent thrombosis after carotid angioplasty and stenting for severe carotid stenosis]. No Shinkei Geka 2007; 35: 1001-1005. (in Japanese)

20) Kobayashi N, Mano K: [Two cases of in-stent thrombosis treated with prasugrel administration: A case report]. NKC 2017; 2: 6-10. (in Japanese)
21) Feher G, Feher A, Pusch G, et al: Clinical importance of aspirin and clopidogrel resistance. World J Cardiol 2010; 2: 171-186.

22) Song TJ, Suh SH, Min PK, et al: The influence of antiplatelet resistance on the development of cerebral ischemic lesion after carotid artery stenting. Yonsei Med J 2013; 54: 288-294.

23) Nakagawa I, Wada T, Park HS, et al: Platelet inhibition by adjunctive cilostazol suppresses the frequency of cerebral ischemic lesions after carotid artery stenting in patients with carotid artery stenosis. J Vasc Surg 2014; 59: 761-767. 